\title{
SOME INEQUALITIES OF ALGEBRAIC POLYNOMIALS HAVING REAL ZEROS
}

\author{
A. K. VARMA
}

Dedicated to Professor A. Zygmund

\begin{abstract}
Let $\boldsymbol{P}_{n}(x)$ be an algebraic polynomial of degree $n$ having all real zeros. We set

$$
I_{n}=\frac{\left\|P_{n}^{\prime}(x) \omega(x)\right\|_{L_{2}[a, b]}}{\left\|P_{n}(x) \omega(x)\right\|_{L_{2}[a, b]}} .
$$

In this work the lower and upper bounds of $I_{n}$ are investigated under the assumptions that all the zeros of $P_{n}(x)$ are inside $[a, b]$ and outside $[a, b]$, respectively. We restrict ourselves here with two cases, (1) $\omega(x)=(1-$ $\left.x^{2}\right)^{1 / 2},[a, b]=[-1,1] ;(2) \omega(x)=e^{-x / 2},[a, b]=[0, \infty)$. Results are shown to be best possible.
\end{abstract}

In this work we are concerned with the following theorems of P. Turán [3], P. Erdős [1], and A. K. Varma [5].

TheOREM A. Let $P_{n}(x)$ be an algebraic polynomial of degree $n$ having all its zeros inside $[-1,+1]$; then we have

$$
\max _{-1<x<+1}\left|P_{n}^{\prime}(x)\right|>\frac{n^{1 / 2}}{6} \max _{-1<x<+1}\left|P_{n}(x)\right| \text {. }
$$

$\left(P_{n}^{\prime}(x)\right.$ stands for the derivative of $P_{n}(x)$.)

THEOREM B. Let $\boldsymbol{P}_{n}(x)$ be a polynomial of degree $n$ satisfying the inequality $\left|P_{n}(x)\right|<1$ for $-1<x<+1$. Suppose $P_{n}(x)$ has only real roots and no root inside $[-1,+1]$; then for $-1<x<+1,\left|P_{n}^{\prime}(x)\right|<\frac{1}{2}$ en. This is the best possible result.

THEOREM C. Let $P_{n}(x)$ be an algebraic polynomial of degree $n$ having all zeros real and inside $[-1,+1]$; then we have

$$
\left\|P_{n}^{\prime}\right\|_{L_{2}[-1,+1]}^{2}>\left(\frac{n}{2}+\frac{3}{4}+\frac{3}{4 n}\right)\left\|p_{n}\right\|_{L_{2}[-1,+1]}^{2}, \quad n>13
$$

where

$$
\left\|P_{n}\right\|_{L_{2}[-1,+1]}^{2}=\int_{-1}^{1} P_{n}^{2}(x) d x .
$$

Further, if $P_{n}(+1)=P_{n}(-1)=0$ and $n \geqslant 2$ then under the above conditions

$$
\left\|P_{n}^{\prime}\right\|_{L_{2}[-1,+1]}^{2} \geqslant\left(\frac{n}{2}+\frac{3}{4}+\frac{3}{4(n-1)}\right)\left\|P_{n}\right\|_{L_{2}[-1,+1]}^{2}
$$

Received by the editors July 26, 1978.

AMS (MOS) subject classifications (1970). Primary 26A60, 30A40. 
with equality holding for $P_{n}(x)=\left(1-x^{2}\right)^{m}, n=2 m$.

The above Theorem $\mathrm{C}$ is analogous to Theorem $\mathrm{A}$ in the $L_{\infty}$ norm. In view of the above theorems it is natural to ask about lower bound (upper bound) of the expression

$$
\left\|\omega(x) P_{n}^{\prime}(x)\right\|_{L_{2}[a, b]} /\left\|\omega(x) P_{n}(x)\right\|_{L_{2}[a, b]}
$$

under the assumption that all the zeros of $P_{n}(x)$ are all inside $[a, b]$ (no roots inside $[a, b]$, respectively). We limit ourselves here in this work to the above question when $[a, b]=[0, \infty), \omega(x)=e^{-x / 2}$ and $[a, b]=[-1,+1], \omega(x)=$ $\left(1-x^{2}\right)^{1 / 2}$.

Throughout $x_{k}$ 's denote the zeros of $P_{n}(x)$. Here we prove the following theorems.

THEOREM 1. Let $P_{n}(x)$ be an algebraic polynomial of degree $n$ having all zeros inside $[0, \infty)$. Let $P_{n}(0)=0$ or

$$
\sum_{k=1}^{n} \frac{1}{x_{k}}>\frac{1}{2}
$$

then

$$
\left\|e^{-x / 2} P_{n}^{\prime}\right\|_{L_{2}(0, \infty)}^{2} \geqslant \frac{n}{2(2 n-1)}\left\|e^{-x / 2} P_{n}\right\|_{L_{2}(0, \infty)}^{2}
$$

with equality for $P_{n}(x)=x^{n}$.

The following remark is needed concerning the above theorem. Let us choose $P_{n}(x)=\left(x-x_{1}\right)^{n}, x_{1}>0$. It can be shown easily that the ratio of

$$
\int_{0}^{\infty}\left(P_{n}^{\prime}(x)\right)^{2} e^{-x} d x / \int_{0}^{\infty}\left(P_{n}(x)\right)^{2} e^{-x} d x
$$

can be made arbitrary small for such polynomials by choosing $x_{1}$ arbitrary large. Thus some condition on the roots such as $\sum_{k=1}^{n}\left(x_{k}\right)^{-1}>\frac{1}{2}$ is natural.

Next, we turn to the case when $\omega(x)=\left(1-x^{2}\right)^{1 / 2},[a, b]=[-1,+1]$. Here we prove

THEOREM 2. Let $P_{n}(x)$ be an algebraic polynomial of degree $n$ having all zeros real and inside $[-1,+1]$. Then for $n>2$ we have

$$
\left\|\left(1-x^{2}\right)^{1 / 2} P_{n}^{\prime}\right\|_{L_{2}[-1,+1]}^{2}>\left(\frac{n}{2}+\frac{1}{4}-\frac{1}{4(n+1)}\right)\left\|\left(1-x^{2}\right)^{1 / 2} P_{n}\right\|_{L_{2}[-1,+1]}^{2}
$$

with equality for $P_{n}(x)=\left(1-x^{2}\right)^{m}, n=2 m$.

The next two theorems are analogous to Theorem B of P. Erdõs [1]. 
THEOREM 3. Let $\boldsymbol{P}_{n}(x)$ be an algebraic polynomial of degree $<n$ having all real roots and no root inside the interval $[-1,+1]$; then we have

$$
\begin{aligned}
\left\|\left(1-x^{2}\right)^{1 / 2} P_{n}^{\prime}(x)\right\|_{L_{2}[-1,+1]}^{2} & \\
& <\frac{n(n+1)(2 n+3)}{4(2 n+1)}\left\|\left(1-x^{2}\right)^{1 / 2} P_{n}(x)\right\|_{L_{2}[-1,+1]}^{2}
\end{aligned}
$$

with equality for $P_{n}(x)=(1+x)^{n}$ or $P_{n}(x)=(1-x)^{n}$.

THEOREM 4. Let $x_{1}, x_{2}, \ldots, x_{n}$ be real zeros of an algebraic polynomial $P_{n}(x)$ of degree $<n$. Further, $-\infty<x_{i}<0, i=1,2, \ldots, n$. Then

$$
\int_{0}^{\infty} x e^{-x}\left(P_{n}^{\prime}(x)\right)^{2} d x<\frac{n}{2(2 n+1)} \int_{0}^{\infty} x e^{-x}\left(P_{n}(x)\right)^{2} d x
$$

with equality for $P_{n}(x)=x^{n}$.

2. Proof of Theorem 1. For the proof of this theorem we will need the following facts:

$$
\begin{gathered}
P_{n}^{\prime}(x)=P_{n}(x) \sum_{k=1}^{n} \frac{1}{x-x_{k}} \\
P_{n}^{\prime 2}(x)-P_{n}(x) P_{n}^{\prime \prime}(x)=P_{n}^{2}(x) \sum_{k=1}^{n} \frac{1}{\left(x-x_{k}\right)^{2}} \\
\frac{\left(x-x_{1}\right)^{2}}{\left(x-x_{k}\right)^{2}}=-1+\frac{2\left(x-x_{1}\right)}{x-x_{k}}+\frac{\left(x_{1}-x_{k}\right)^{2}}{\left(x-x_{k}\right)^{2}} \\
P_{n}^{\prime}(x)-n \frac{P_{n}(x)}{x-x_{1}}=\frac{P_{n}(x)}{x-x_{1}} \sum_{k=1}^{n} \frac{x_{k}-x_{1}}{x-x_{k}}
\end{gathered}
$$

From (2.4) and the Cauchy-Schwarz inequality we have

$$
\left(P_{n}^{\prime}(x)-n \frac{P_{n}(x)}{x-x_{1}}\right)^{2} \leqslant n \frac{P_{n}^{2}(x)}{\left(x-x_{1}\right)^{2}} \sum_{k=1}^{n} \frac{\left(x_{k}-x_{1}\right)^{2}}{\left(x-x_{k}\right)^{2}}
$$

From (2.5) we immediately have

$$
\begin{aligned}
\int_{0}^{\infty}\left(P_{n}^{\prime}(x)-n \frac{P_{n}(x)}{x-x_{1}}\right)^{2} e^{-x} d x & \\
& <n \int_{0}^{\infty} \frac{P_{n}^{2}(x)}{\left(x-x_{1}\right)^{2}} \sum_{k=1}^{n} \frac{\left(x_{k}-x_{1}\right)^{2}}{\left(x-x_{k}\right)^{2}} e^{-x} d x
\end{aligned}
$$

Also, integrating by parts, we have

$$
\int_{0}^{\infty} e^{-x} P_{n}(x) P_{n}^{\prime}(x) d x=-\frac{1}{2} P_{n}^{2}(0)+\frac{1}{2} \int_{0}^{\infty} e^{-x} P_{n}^{2}(x) d x .
$$


Now, we turn to the proof of Theorem 1. On integrating by parts, we have

$$
\int_{0}^{\infty} e^{-x} P_{n}^{\prime 2}(x) d x=-P_{n}(0) P_{n}^{\prime}(0)-\int_{0}^{\infty} P_{n}(x)\left(P_{n}^{\prime \prime}(x)-P_{n}^{\prime}(x)\right) e^{-x} d x
$$

and an equivalent form

$$
\begin{aligned}
2 \int_{0}^{\infty} e^{-x} P_{n}^{\prime 2}(x) d x= & P_{n}^{2}(0) \sum_{k=1}^{n} \frac{1}{x_{k}}+\int_{0}^{\infty} e^{-x}\left(P_{n}^{\prime 2}(x)-P_{n}(x) P_{n}^{\prime \prime}(x)\right) d x \\
& +\int_{0}^{\infty} e^{-x} P_{n}(x) P_{n}^{\prime}(x) d x
\end{aligned}
$$

On using (2.2) and (2.7) we obtain

$$
\begin{aligned}
2 \int_{0}^{\infty} e^{-x} P_{n}^{\prime 2}(x) d x= & P_{n}^{2}(0)\left(-\frac{1}{2}+\sum_{k=1}^{n} \frac{1}{x_{k}}\right)+\frac{1}{2} \int_{0}^{\infty} e^{-x} P_{n}^{2}(x) d x \\
& +\int_{0}^{\infty} e^{-x} P_{n}^{2}(x) \sum_{k=1}^{n} \frac{1}{\left(x-x_{k}\right)^{2}} d x
\end{aligned}
$$

Now, let us denote by $x_{1}$ the first nonnegative zero of $P_{n}(x)$. Then we can write

$$
P_{n}(x)=\left(x-x_{1}\right) q_{n-1}(x), \quad x_{1}>0
$$

where $q_{n-1}(x)$ is a polynomial of degree $<n-1$ having real zeros lying inside $[0, \infty)$.

We set

$$
I_{1}=\int_{0}^{\infty} e^{-x} P_{n}^{2}(x) \sum_{k=1}^{n} \frac{1}{\left(x-x_{k}\right)^{2}} d x=\int_{0}^{\infty} e^{-x} q_{n-1}^{2}(x) \sum_{k=1}^{n} \frac{\left(x-x_{1}\right)^{2}}{\left(x-x_{k}\right)^{2}} d x,
$$

and on using (2.3) we have

$$
\begin{aligned}
I_{1}= & -n \int_{0}^{\infty} e^{-x} q_{n-1}^{2}(x) d x+2 \int_{0}^{\infty}\left(x-x_{1}\right) q_{n-1}^{2}(x) \sum_{k=1}^{n} \frac{1}{\left(x-x_{k}\right)} e^{-x} d x \\
& +\int_{0}^{\infty} e^{-x} q_{n-1}^{2}(x) \sum_{k=1}^{n} \frac{\left(x_{1}-x_{k}\right)^{2}}{\left(x-x_{k}\right)^{2}} d x \\
= & -n \int_{0}^{\infty} e^{-x} q_{n-1}^{2}(x) d x+2 \int_{0}^{\infty} P_{n}^{\prime}(x) q_{n-1}(x) e^{-x} d x \\
& +\int_{0}^{\infty} e^{-x} q_{n-1}^{2}(x) \sum_{k=1}^{n} \frac{\left(x_{1}-x_{k}\right)^{2}}{\left(x-x_{k}\right)^{2}} d x \\
I_{1}= & \frac{1}{n} \int_{0}^{\infty} P_{n}^{\prime 2}(x) e^{-x} d x-\frac{1}{n} \int_{0}^{\infty}\left(P_{n}^{\prime}(x)-n q_{n-1}(x)\right)^{2} e^{-x} d x \\
& +\int_{0}^{\infty} e^{-x} q_{n-1}^{2}(x) \sum_{k=1}^{n} \frac{\left(x_{1}-x_{k}\right)^{2}}{\left(x-x_{k}\right)^{2}} d x
\end{aligned}
$$


on putting the values of $I_{1}$ from (2.13) into (2.10) we obtain

$$
\begin{aligned}
\left(2-\frac{1}{n}\right) \int_{0}^{\infty} e^{-x} P_{n}^{\prime 2}(x) d x= & P_{n}^{2}(0)\left(-\frac{1}{2}+\sum_{k=1}^{n} \frac{1}{x_{k}}\right)+\frac{1}{2} \int_{0}^{\infty} e^{-x} P_{n}^{2}(x) d x \\
& +\int_{0}^{\infty} e^{-x} q_{n-1}^{2}(x) \sum_{k=1}^{n} \frac{\left(x_{1}-x_{k}\right)^{2}}{\left(x-x_{k}\right)^{2}} d x \\
& -\frac{1}{n} \int_{0}^{\infty}\left(P_{n}^{\prime}(x)-n q_{n-1}(x)\right)^{2} e^{-x} d x
\end{aligned}
$$

Now if $P_{n}(0)=0$ or $\sum_{k=1}^{n}\left(x_{k}\right)^{-1}>\frac{1}{2}$ then on using (2.6) we immediately obtain

$$
\left(2-\frac{1}{n}\right) \int_{0}^{\infty} e^{-x} P_{n}^{\prime 2}(x) d x>\frac{1}{2} \int_{0}^{\infty} e^{-x} P_{n}^{2}(x) d x,
$$

from which the proof of Theorem 1 is complete. We would like to remark that the ratio of

$$
\int_{0}^{\infty} e^{-x} P_{n}^{\prime 2}(x) d x / \int_{0}^{\infty} e^{-x} P_{n}^{2}(x) d x
$$

does indeed get smaller than $n / 2(2 n-1)$ for polynomials $P_{0}(x)=\left(x-x_{1}\right)^{n}$ for $x_{1}>2 n$. For such polynomials the last two terms in (2.14) are zero and $-\frac{1}{2}+\sum_{k=1}^{n}\left(x_{k}\right)^{-1}<0$.

3. Proof of Theorem 2. In order to prove Theorem 2, we need some results proved in [5]. Let $P_{n}(x)$ be any polynomial of degree $n$; then

$$
\begin{gathered}
2 \int_{-1}^{1}\left(1-x^{2}\right) P_{n}^{\prime 2}(x) d x=n \int_{-1}^{1} P_{n}^{2}(x) d x+S_{0} \\
(n+1)(2 n+3) \int_{-1}^{1} x^{2} P_{n}^{2}(x) d x=S_{2}-S_{0}+(n+1) \int_{-1}^{1} P_{n}^{2}(x) d x \\
+2 \int_{-1}^{1}\left(\left(1-x^{2}\right) P_{n}^{\prime}(x)+n x P_{n}(x)\right)^{2} d x
\end{gathered}
$$

where

$$
S_{2 i}=\int_{-1}^{1} x^{2 i} P_{n}^{2}(x) \sum_{k=1}^{n} \frac{\left(1-x_{k}^{2}\right)}{\left(x-x_{k}\right)^{2}} d x, \quad i=0,1, \ldots
$$

For the proof of (3.1), (3.2) see (2.1) and (2.10) (with $r=1$ ) in [5]. Equation (3.2) can be rewritten in the form

$$
\begin{aligned}
(n+1)(2 n+3) & \int_{-1}^{1}\left(1-x^{2}\right) P_{n}^{2}(x) d x \\
= & S_{0}-S_{2}+2(n+1)^{2} \int_{-1}^{1} P_{n}^{2}(x) d x \\
& -2 \int_{-1}^{1}\left(\left(1-x^{2}\right) P_{n}^{\prime}(x)+n x P_{n}(x)\right)^{2} d x
\end{aligned}
$$


Therefore

$$
(n+1)(2 n+3) \int_{-1}^{1}\left(1-x^{2}\right) P_{n}^{2}(x) d x<S_{0}-S_{2}+2(n+1)^{2} \int_{-1}^{1} P_{n}^{2}(x) d x
$$

From (3.1) and (3.4) we obtain

$$
\frac{2 \int_{-1}^{1}\left(1-x^{2}\right) P_{n}^{\prime 2}(x) d x}{(n+1)(2 n+3) \int_{-1}^{1}\left(1-x^{2}\right) P_{n}^{2}(x) d x}>\frac{n \int_{-1}^{1} P_{n}^{2}(x) d x+S_{0}}{S_{0}-S_{2}+2(n+1)^{2} \int_{-1}^{1} P_{n}^{2}(x) d x} .
$$

It is given that $\left|x_{k}\right|<1, S_{0}>0, S_{2}>0$ and $S_{0}-S_{2}>0$ (here we use (3.3)). Hence it can be verified immediately that

$$
\begin{aligned}
\left(S_{0}+n \int_{-1}^{1} P_{n}^{2}(x) d x\right) & /\left(S_{0}-S_{2}+2(n+1)^{2} \int_{-1}^{1} P_{n}^{2}(x) d x\right) \\
& >n / 2(n+1)^{2} .
\end{aligned}
$$

From (3.5) and (3.6) we obtain

$$
\int_{-1}^{1}\left(1-x^{2}\right) P_{n}^{\prime 2}(x) d x>\frac{n(2 n+3)}{4(n+1)} \int_{-1}^{1}\left(1-x^{2}\right) P_{n}^{2}(x) d x .
$$

This proves Theorem 2 as well.

4. Proof of Theorem 3. For the proof of Theorem 3 we need the following lemma.

LEMMA 4.1. Let $P_{n}(x)$ be an algebraic polynomial of degree $<n$ having real roots and no root in the interval $[-1,+1]$. Then we have

$$
\int_{-1}^{1}\left(1-x^{2}\right) P_{n}^{2}(x) d x>\frac{2(2 n+1)}{(n+1)(2 n+3)} \int_{-1}^{1} P_{n}^{2}(x) d x
$$

Proof It follows from the well-known theorem [2] that for such $P_{n}(x)$ we have

$$
P_{n}^{2}(x)=\sum_{p+q=2 n} a_{p q}(1+x)^{p}(1-x)^{q}, \quad a_{p q}>0
$$

Hence we have

$$
\int_{-1}^{1}\left(1-x^{2}\right) P_{n}^{2}(x) d x=\sum_{p+q=2 n} a_{p q} \int_{-1}^{1}(1+x)^{p+1}(1-x)^{q+1} d x .
$$


But

$$
\begin{aligned}
& \int_{-1}^{1}(1+x)^{p+1}(1-x)^{q+1} d x \\
& =\frac{4(p+1)(q+1)}{(p+q+3)(p+q+2)} \int_{-1}^{1}(1+x)^{p}(1-x)^{q} d x
\end{aligned}
$$

Hence

$$
\begin{aligned}
\int_{-1}^{1}\left(1-x^{2}\right) P_{n}^{2}(x) d x & \\
& =4 \sum_{p+q=2 n} \frac{a_{p q}(p+1)(q+1)}{(p+q+3)(p+q+2)} \int_{-1}^{1}(1+x)^{p}(1-x)^{q} d x \\
& >\frac{4(2 n+1)}{(2 n+3)(2 n+2)} \sum_{p+q=2 n} q_{p q} \int_{-1}^{1}(1+x)^{p}(1-x)^{q} d x \\
& =\frac{2(2 n+1)}{(n+1)(2 n+3)} \int_{-1}^{1} P_{n}^{2}(x) d x
\end{aligned}
$$

This proves the lemma. Now we turn to the proof of the theorem. Since $\left|x_{k}\right|>1, k=1,2, \ldots, n$, it follows from (3.1) and (3.3) that

$$
2 \int_{-1}^{1}\left(1-x^{2}\right) P_{n}^{\prime 2}(x) d x<n \int_{-1}^{1} P_{n}^{2}(x) d x,
$$

with equality holding for $P_{n}(x)=(1+x)^{p}(1-x)^{q}, p+q=n$. Now, on using (4.1) and (4.3) we obtain

$$
\begin{aligned}
\frac{\int_{-1}^{1}\left(1-x^{2}\right) P_{n}^{\prime 2}(x) d x}{\int_{-1}^{1}\left(1-x^{2}\right) P_{n}^{2}(x) d x} & =\frac{\int_{-1}^{1}\left(1-x^{2}\right) P_{n}^{\prime 2}(x) d x}{\int_{-1}^{1} P_{n}^{2}(x) d x} \frac{\int_{-1}^{1} P_{n}^{2}(x) d x}{\int_{-1}^{1}\left(1-x^{2}\right) P_{n}^{2}(x) d x} \\
& <\frac{n}{2} \cdot \frac{(n+1)(2 n+3)}{2(2 n+1)}
\end{aligned}
$$

This completes the proof of Theorem 3.

5. Proof of Theorem 4. On integrating by parts

$$
\begin{aligned}
\int_{0}^{\infty} P_{n}^{\prime \prime}(x) P_{n}(x) e^{-x} x d x= & -\int_{0}^{\infty} P_{n}^{\prime}(x)\left\{P_{n}^{\prime}(x) x+P_{n}(x)-x P_{n}(x)\right\} e^{-x} d x \\
= & -\int_{0}^{\infty} x e^{-x}\left(P_{n}^{\prime}(x)\right)^{2} d x+\frac{1}{2} P_{n}^{2}(0) \\
& +\frac{1}{2} \int_{0}^{\infty} x e^{-x} P_{n}^{2}(x) d x-\int_{0}^{\infty} e^{-x} P_{n}^{2}(x) d x
\end{aligned}
$$


Since $x_{k}<0, k=1,2, \ldots, n$, we have

$$
\begin{aligned}
\int_{0}^{\infty} x e^{-x} P_{n}^{2}(x) \sum_{k=1}^{n} \frac{1}{\left(x-x_{k}\right)^{2}} d x & <\int_{0}^{\infty} e^{-x} P_{n}^{2}(x) \sum_{k=1}^{n} \frac{1}{x-x_{k}} d x \\
& =\int_{0}^{\infty} e^{-x} P_{n}(x) P_{n}^{\prime}(x) d x \\
& =-\frac{1}{2} P_{n}^{2}(0)+\frac{1}{2} \int_{0}^{\infty} P_{n}^{2}(x) e^{-x} d x
\end{aligned}
$$

On using (5.1) and (5.2) and the identity

$$
P_{n}^{\prime 2}(x)=P_{n}(x) P_{n}^{\prime \prime}(x)+P_{n}^{2}(x) \sum_{k=1}^{n} \frac{1}{\left(x-x_{k}\right)^{2}}
$$

we obtain

$$
2 \int_{0}^{\infty} P_{n}^{\prime 2}(x) x e^{-x} d x<\frac{1}{2} \int_{0}^{\infty} x e^{-x} P_{n}^{2}(x) d x-\frac{1}{2} \int_{0}^{\infty} e^{-x} P_{n}^{2}(x) d x .
$$

It is easy to see that under the conditions of our theorem

Hence

$$
P_{n}^{2}(x)=\sum_{k=0}^{2 n} a_{k} x^{k}, \quad a_{k}>0 .
$$

$$
\int_{0}^{\infty} e^{-x} P_{n}^{2}(x) d x=\sum_{k=0}^{2 n} a_{k} \int_{0}^{\infty} e^{-x} x^{k} d x=\sum_{k=0}^{2 n} a_{k} k !
$$

similarly

$$
\begin{aligned}
\int_{0}^{\infty} e^{-x} x P_{n}^{2}(x) d x & =\sum_{k=0}^{2 n} a_{k}(k+1) ! \\
& <(2 n+1) \sum_{k=0}^{2 n} a_{k} k !=(2 n+1) \int_{0}^{\infty} e^{-x} P_{n}^{2}(x) d x
\end{aligned}
$$

On using (5.3) and the above result it follows that

$$
2 \int_{0}^{\infty}\left(P_{n}^{\prime}(x)\right)^{2} x e^{-x} d x<\left(\frac{1}{2}-\frac{1}{(2 n+1) 2}\right) \int_{0}^{\infty} P_{n}^{2}(x) x e^{-x} d x .
$$

From this Theorem 4 now follows.

\section{REFERENCES}

1. P. Erdös, On extremal properties of the derivatives of polynomials, Ann. of Math. (2) 41 (1941), 310-313.

2. G. G. Lorentz, The degree of approximation by polynomials with pasitive coefficients, Math. Ann. 151 (1963) 239-251.

3. P. Turán, Über die Ableitung von Polynomen, Compositio Math. 7 (1939), 89-95.

4. A. K. Varma, An analogue of some inequalities of P. Turan, Proc. Amer. Math. Soc. 65 (1976), 305-309.

5. , An analogue of some inequalities of P. Turán. II, Proc. Amer. Math. Soc. $\mathscr{Q}$ (1978), 25-33.

Department of Mathematics, University of Florida, Gainesville, Florma 32611 